\title{
A Class of Estimators for Finite Population Mean in Double Sampling under Nonresponse Using Fractional Raw Moments
}

\author{
Manzoor Khan, ${ }^{1}$ Javid Shabbir, ${ }^{1}$ Zawar Hussain, ${ }^{1}$ and Bander Al-Zahrani ${ }^{2}$ \\ ${ }^{1}$ Department of Statistics, Quaid-i-Azam University, Islamabad 45320, Pakistan \\ ${ }^{2}$ Department of Statistics, King Abdulaziz University, P.O. Box 80203, Jeddah 21589, Saudi Arabia
}

Correspondence should be addressed to Zawar Hussain; zhlangah@yahoo.com

Received 11 May 2014; Revised 18 July 2014; Accepted 21 July 2014; Published 18 August 2014

Academic Editor: Boris Andrievsky

Copyright (C) 2014 Manzoor Khan et al. This is an open access article distributed under the Creative Commons Attribution License, which permits unrestricted use, distribution, and reproduction in any medium, provided the original work is properly cited.

\begin{abstract}
This paper presents new classes of estimators in estimating the finite population mean under double sampling in the presence of nonresponse when using information on fractional raw moments. The expressions for mean square error of the proposed classes of estimators are derived up to the first degree of approximation. It is shown that a proposed class of estimators performs better than the usual mean estimator, ratio type estimators, and Singh and Kumar (2009) estimator. An empirical study is carried out to demonstrate the performance of a proposed class of estimators.
\end{abstract}

\section{Introduction}

Generally almost all surveys suffer from the problem of nonresponse. Lack of information, absence at the time of survey, and refusal of the respondent are the main causes of nonresponse. Hansen and Hurwitz [1] suggested a procedure of taking a subsample from the nonrespondent and collecting information by a more expensive method like first attempt by mail questionnaire and second attempt by personal interview. In estimation of population parameters like mean, total, and ratio, the auxiliary information is some time incorporated with the study variable to improve the efficiency of the estimators. Cochran [2], Rao [3, 4], Khare and Srivastava [57], Okafor and Lee [8], Tabasum and Khan [9, 10], Sodipo and Obisesan [11], Singh and Kumar [12-14], and Singh et al. [15] have studied the problem of nonresponse under double sampling using the auxiliary information. Dubey and Uprety [16] used second raw moments for efficient estimation of population means in the presence of nonresponse. AlHossain and Khan [17] used maximum and minimum values of population for improved estimation of population mean.

In double sampling, a large sample of size say $n^{\prime}$ is selected by simple random sample without replacement (SRSWOR) sampling scheme, at first phase from a population $U=$ $\left(U_{1}, U_{2}, \ldots, U_{N}\right)$, and then a smaller sample of size say $n$ (that is $n<n^{\prime}$ ) is also selected by SRSWOR at the second phase or directly from $U=\left(U_{1}, U_{2}, \ldots, U_{N}\right)$. Nonresponse occurs on the second phase sample of size $n$ in which $n_{1}$ units respond and $n_{2}$ units do not. From the $n_{2}$ nonrespondents, by SRSWOR a subsample of $r=n_{2} / k ; k>1$ units is selected, where $k$ is the inverse sampling rate at the second phase sample of size $n$. All the $r$ units are assumed to respond this time round. The auxiliary information can be used at the estimation stage to compensate for units selected for the sample that fail to provide adequate responses and for population units missing from the sampling frame. For example, in a manufacturing survey, the number of labourers can be used as an auxiliary variable for the estimation of items produced in bales. Information can be obtained completely on the number of labourers while there may be nonresponse on the amount of item produced in bales.

Hansen and Hurwitz [1] proposed an unbiased estimator of the population mean $(\bar{Y})$ given by

$$
\bar{y}^{*}=w_{1} \bar{y}_{1}+w_{2} \bar{y}_{2 r}
$$

where $w_{i}=n_{i} / n$ for $i=1,2 ; \bar{y}_{1}=\sum_{i=1}^{n_{1}} y_{i} / n_{1}$ and $\bar{y}_{2 r}=$ $\sum_{i=1}^{r} y_{i} / r$. 
The variance of unbiased estimator $\left(\bar{y}^{*}\right)$ is given by

$$
V\left(\bar{y}^{*}\right)=\left(\frac{1}{n}-\frac{1}{N}\right) S_{y}^{2}+\frac{W_{2}(k-1) S_{y(2)}^{2}}{n},
$$

where $S_{y}^{2}=\sum_{i=1}^{N}\left(y_{i}-\bar{Y}\right)^{2} /(N-1), S_{y(2)}^{2}=\sum_{i=1}^{N_{2}}\left(y_{i}-\bar{Y}_{2}\right)^{2} /\left(N_{2}-\right.$ 1 ), and $W_{2}=N_{2} / N$.

Let $\bar{Y}_{1}=\sum_{i=1}^{N_{1}} y_{i} / N_{1}$ and $\bar{Y}_{2}=\sum_{i=1}^{N_{2}} y_{i} / N_{2}$ be the population means of respondents and nonrespondents, respectively, and let $\bar{Y}=\sum_{i=1}^{N} y_{i} / N$ be the mean of whole population mean.

Generally the auxiliary information can be used to increase the precision of the estimators for estimating the population mean $(\bar{Y})$. Auxiliary information can be transformed to enhance the precision of estimation even further. Let us denote the auxiliary information by variable $x$ and a transformation of the auxiliary variable in the form of raw moments by $u=x^{p}$, where $p>0$. Further, we assume that $x>0$. The variables $x$ and $u$ take values $x_{i}$ and $u_{i}$, $(i=1,2, \ldots, N)$ on $i$ th unit of population.

Unbiased estimators for the population mean $\bar{X}=$ $\sum_{i=1}^{N} x_{i} / N$ and $p$ th raw moments $\bar{U}=\sum_{i=1}^{N} x_{i}^{p} / N$ of the population are given by

$$
\begin{aligned}
& \bar{x}^{*}=w_{1} \bar{x}_{1}+w_{2} \bar{x}_{2 r}, \\
& \bar{u}^{*}=w_{1} \bar{u}_{1}+w_{2} \bar{u}_{2 r},
\end{aligned}
$$

where $\bar{x}_{1}=\sum_{i=1}^{n_{1}} x_{i} / n_{1}, \bar{x}_{2 r}=\sum_{i=1}^{r} x_{i} / r, \bar{u}_{1}=\sum_{i=1}^{n_{1}} x_{i}^{p} / n_{1}$, and $\bar{u}_{2 r}=\sum_{i=1}^{r} x_{i}^{p} / r$ are the sample means and sample $p$ th raw moments of respondents and nonrespondents of population based on a sample of size $n_{1}$ and a subsample of size $r=$ $n_{2} / k$. The corresponding population means and population $p$ th raw moments of the respondents and nonrespondents are given by $\bar{X}_{1}=\sum_{i=1}^{N_{1}} x_{i} / N_{1}, \bar{X}_{2}=\sum_{i=1}^{N_{2}} x_{i} / N_{2}, \bar{U}_{1}=$ $\sum_{i=1}^{N_{1}} x_{i}^{p} / N_{1}$, and $\bar{U}_{2}=\sum_{i=1}^{N_{2}} x_{i}^{p} / N_{2}$, respectively.

Now the variances of $\bar{x}^{*}$ and $\bar{u}^{*}$ are given by

$$
V\left(\bar{x}^{*}\right)=\left(\frac{1}{n}-\frac{1}{N}\right) S_{x}^{2}+\frac{W_{2}(k-1) S_{x(2)}^{2}}{n},
$$

where $S_{x}^{2}=\sum_{i=1}^{N}\left(x_{i}-\bar{X}\right)^{2} /(N-1)$ and $S_{x(2)}^{2}=\sum_{i=1}^{N_{2}}\left(x_{i}-\right.$ $\left.\bar{X}_{2}\right)^{2} /\left(N_{2}-1\right)$ and

$$
V\left(\bar{u}^{*}\right)=\left(\frac{1}{n}-\frac{1}{N}\right) S_{u}^{2}+\frac{W_{2}(k-1) S_{u(2)}^{2}}{n},
$$

where $S_{u}^{2}=\sum_{i=1}^{N}\left(u_{i}-\bar{U}\right)^{2} /(N-1)$ and $S_{u(2)}^{2}=\sum_{i=1}^{N_{2}}\left(u_{i}-\right.$ $\left.\bar{U}_{2}\right)^{2} /\left(N_{2}-1\right)$.

Now we define the relative error terms.

Let

$$
\begin{gathered}
e_{0}^{*}=\frac{\bar{y}^{*}-\bar{Y}}{\bar{Y}}, \quad e_{1}^{*}=\frac{\bar{x}^{*}-\bar{X}}{\bar{X}}, \\
e_{1}=\frac{\bar{x}-\bar{X}}{\bar{X}}, \quad e_{1}^{\prime}=\frac{\bar{x}^{\prime}-\bar{X}}{\bar{X}}, \\
e_{2}^{*}=\frac{\bar{u}^{*}-\bar{U}}{\bar{U}}, \quad e_{2}=\frac{\bar{u}-\bar{U}}{\bar{U}}, \\
e_{2}^{\prime}=\frac{\bar{u}^{\prime}-\bar{U}}{\bar{U}},
\end{gathered}
$$

such that $E\left(e_{0}^{*}\right)=E\left(e_{i}^{*}\right)=E\left(e_{i}\right)=E\left(e_{i}^{\prime}\right)=0, i=1,2$ :

$$
\begin{aligned}
& E\left(e_{0}^{* 2}\right)=\lambda C_{y}^{2}+\lambda^{*} C_{y(2)}^{2}, \\
& E\left(e_{1}^{* 2}\right)=\lambda C_{x}^{2}+\lambda^{*} C_{x(2)}^{2}, \\
& E\left(e_{1}^{2}\right)=\lambda C_{x}^{2}, \quad E\left(e_{1}^{\prime 2}\right)=\lambda^{\prime} C_{x}^{2}, \\
& E\left(e_{2}^{* 2}\right)=\lambda C_{u}^{2}+\lambda^{*} C_{u(2)}^{2}, \\
& E\left(e_{2}^{2}\right)=\lambda C_{u}^{2}, \quad E\left(e_{2}^{\prime 2}\right)=\lambda^{\prime} C_{u}^{2}, \\
& E\left(e_{0}^{*} e_{1}^{*}\right)=\lambda \rho_{y x} C_{y} C_{x}+\lambda^{*} \rho_{y x(2)} C_{y(2)} C_{x(2)} \text {, } \\
& E\left(e_{0}^{*} e_{2}^{*}\right)=\lambda \rho_{y u} C_{y} C_{u}+\lambda^{*} \rho_{y u(2)} C_{y(2)} C_{u(2)} \text {, } \\
& E\left(e_{0}^{*} e_{1}^{\prime}\right)=\lambda^{\prime} \rho_{y x} C_{y} C_{x}, \\
& E\left(e_{0}^{*} e_{1}\right)=\lambda \rho_{y x} C_{y} C_{x}, \\
& E\left(e_{0}^{*} e_{2}^{\prime}\right)=\lambda^{\prime} \rho_{y u} C_{y} C_{u} \text {, } \\
& E\left(e_{0}^{*} e_{2}\right)=\lambda \rho_{y u} C_{y} C_{u} \text {, } \\
& E\left(e_{1}^{*} e_{1}\right)=\lambda C_{x}^{2}, \quad E\left(e_{1}^{*} e_{1}^{\prime}\right)=\lambda^{\prime} C_{x}^{2}, \\
& E\left(e_{1} e_{1}^{\prime}\right)=\lambda^{\prime} C_{x}^{2}, \quad E\left(e_{2}^{*} e_{2}\right)=\lambda C_{u}^{2}, \\
& E\left(e_{2}^{*} e_{2}^{\prime}\right)=\lambda^{\prime} C_{u}^{2}, \quad E\left(e_{2} e_{2}^{\prime}\right)=\lambda^{\prime} C_{u}^{2}, \\
& E\left(e_{1}^{*} e_{2}^{\prime}\right)=\lambda^{\prime} \rho_{x u} C_{x} C_{u}, \\
& E\left(e_{1}^{*} e_{2}^{*}\right)=\lambda \rho_{x u} C_{x} C_{u}+\lambda^{*} \rho_{x u(2)} C_{x(2)} C_{u(2)}, \\
& E\left(e_{1}^{*} e_{2}\right)=\lambda \rho_{x u} C_{x} C_{u}, \quad E\left(e_{1} e_{2}^{*}\right)=\lambda \rho_{x u} C_{x} C_{u} \text {, } \\
& E\left(e_{1} e_{2}^{\prime}\right)=\lambda^{\prime} \rho_{x u} C_{x} C_{u}, \\
& E\left(e_{1} e_{2}\right)=\lambda \rho_{x u} C_{x} C_{u}, \\
& E\left(e_{1}^{\prime} e_{2}^{*}\right)=\lambda^{\prime} \rho_{x u} C_{x} C_{u} \text {, } \\
& E\left(e_{1}^{\prime} e_{2}^{\prime}\right)=\lambda^{\prime} \rho_{x u} C_{x} C_{u} \text {, } \\
& E\left(e_{1}^{\prime} e_{2}\right)=\lambda^{\prime} \rho_{x u} C_{x} C_{u}, \\
& \lambda=\frac{(1-f)}{n}, \quad \lambda^{\prime}=\frac{\left(1-f^{\prime}\right)}{n^{\prime}} \text {, } \\
& \lambda^{*}=\frac{w_{2}(k-1)}{n^{\prime}}, \\
& f=\frac{n}{N}, \quad f^{\prime}=\frac{n^{\prime}}{N} \text {. }
\end{aligned}
$$

Here $\left(\rho_{y x}, \rho_{y u}, \rho_{x u}\right)$ and $\left(\rho_{y x(2)}, \rho_{y x(2)}, \rho_{x u(2)}\right)$ are, respectively, the correlation coefficients of respondent and nonrespondent groups to their respective subscripts. Let $\left(C_{y}, C_{x}, C_{u}\right)$ and $\left(C_{y(2)}, C_{x(2)}, C_{u(2)}\right)$ be the coefficients of variation of their 
respective subscripts for the respondent and nonrespondent groups, respectively. Similarly we define

$$
\begin{aligned}
& k_{y x}=\frac{\beta_{y x}}{R_{1}}, \quad k_{y x(2)}=\frac{\beta_{y x(2)}}{R_{1}}, \\
& k_{y u}=\frac{\beta_{y u}}{R_{2}}, \quad k_{y u(2)}=\frac{\beta_{y u(2)}}{R_{2}}, \\
& k_{x u}=\frac{\beta_{x u}}{R_{3}}, \quad k_{x u(2)}=\frac{\beta_{x u(2)}}{R_{3}}, \\
& R_{1}=\frac{\bar{Y}}{\bar{X}}, \quad R_{2}=\frac{\bar{Y}}{\bar{U}}, \quad R_{3}=\frac{\bar{X}}{\bar{U}}, \\
& \beta_{y x}=\frac{\sum_{i=1}^{N}\left(y_{i}-\bar{Y}\right)\left(x_{i}-\bar{X}\right)}{\sum_{i=1}^{N}\left(x_{i}-\bar{X}\right)^{2}}, \\
& \beta_{y x(2)}=\frac{\sum_{i=1}^{N_{2}}\left(y_{i}-\bar{Y}_{2}\right)\left(x_{i}-\bar{X}_{2}\right)}{\sum_{i=1}^{N_{2}}\left(x_{i}-\bar{X}_{2}\right)^{2}}, \\
& \beta_{y u}=\frac{\sum_{i=1}^{N}\left(u_{i}-\bar{U}\right)\left(y_{i}-\bar{Y}\right)}{\sum_{i=1}^{N}\left(u_{i}-\bar{U}\right)^{2}}, \\
& \beta_{y u(2)}=\frac{\sum_{i=1}^{N_{2}}\left(u_{i}-\bar{U}_{2}\right)\left(y_{i}-\bar{Y}_{2}\right)}{\sum_{i=1}^{N_{2}}\left(u_{i}-\bar{U}_{2}\right)^{2}}, \\
& \beta_{x u}=\frac{\sum_{i=1}^{N}\left(x_{i}-\bar{X}\right)\left(u_{i}-\bar{U}\right)}{\sum_{i=1}^{N}\left(u_{i}-\bar{U}\right)^{2}}, \\
& \beta_{x u(2)}=\frac{\sum_{i=1}^{N_{2}}\left(x_{i}-\bar{X}_{2}\right)\left(u_{i}-\bar{U}_{2}\right)}{\sum_{i=1}^{N_{2}}\left(u_{i}-\bar{U}_{2}\right)^{2}} .
\end{aligned}
$$

\section{A Proposed Class of Estimators}

We propose a general class of estimators which utilizes pth raw moments along with auxiliary information under double sampling scheme. The estimator covers all situations of nonresponse; for instance, there may be nonresponse on both the study variable $(y)$ and the auxiliary variable $(x)$ and in other cases there may be complete response on the auxiliary variable and incomplete response on study variable.

Singh and Kumar [13] proposed the following class of estimators:

$$
\widehat{\bar{Y}}_{\mathrm{SK}}=\bar{y}^{*}\left(\frac{A \bar{x}^{*}+B}{A \bar{x}^{\prime}+B}\right)^{\alpha}\left(\frac{A \bar{x}+B}{A \bar{x}^{\prime}+B}\right)^{\beta} \text {, }
$$

where $\bar{x}=\sum_{i=1}^{n} x_{i} / n, \bar{x}^{\prime}=\sum_{i=1}^{n^{\prime}} x_{i} / n^{\prime}, A$ and $B$ are population parameters, and $\alpha$ and $\beta$ are constants to be determined and are selected so that mean square error of $\hat{\bar{Y}}_{\mathrm{SK}}$ becomes minimum. The bias $(B)$ and mean square error $(M)$ of $\widehat{\bar{Y}}_{\mathrm{SK}}$, to the first degree of approximation, are, respectively, given by

$$
\begin{aligned}
B\left(\hat{\bar{Y}}_{\mathrm{SK}}\right)=\bar{Y}[ & \left(\lambda-\lambda^{\prime}\right) \phi \\
& \times\left(\alpha\left(k_{y x}+\frac{\alpha-1}{2} \phi\right)\right. \\
& \left.+\beta\left(k_{y x}+\alpha \phi+\frac{\beta-1}{2} \phi\right)\right) C_{x}^{2} \\
& \left.+\lambda^{*}\left(\alpha \phi\left(k_{y x(2)}+\frac{\alpha-1}{2} \phi\right) C_{x(2)}^{2}\right)\right], \\
M\left(\hat{\bar{Y}}_{\mathrm{SK}}\right)=\bar{Y}^{2}[ & \left(\lambda-\lambda^{\prime}\right) \\
& \times\left\{C_{y}^{2}+(\alpha+\beta) \phi\left((\alpha+\beta) \phi+2 k_{y x}\right) C_{x}^{2}\right\} \\
& +\lambda^{*}\left\{C_{y(2)}^{2}+\alpha \phi\left(\alpha \phi+2 k_{y x(2)}\right) C_{x(2)}^{2}\right\} \\
& \left.+\lambda^{\prime} C_{y}^{2}\right],
\end{aligned}
$$

where $\phi=(A \bar{X} /(A \bar{X}+B))$.

The mean square error of $\widehat{\bar{Y}}_{\mathrm{SK}}$ for optimum values of $\alpha$ and $\beta$, that is, $\alpha=\left(\left(k_{y x(2)}-k_{y x}\right) / \phi\right)$ and $\beta=-\left(k_{y x(2)} / \phi\right)$, is given by

$$
\begin{aligned}
M\left(\hat{\bar{Y}}_{\mathrm{SK}}\right)_{\mathrm{opt}} \cong \bar{Y}^{2}[ & \left(\lambda-\lambda^{\prime}\right) C_{y}^{2}\left\{1-\rho_{y x}^{2}\right\} \\
& \left.+\lambda^{*} C_{y}^{2}\left\{1-\rho_{y x(2)}^{2}\right\}+\lambda^{\prime} C_{y}^{2}\right] .
\end{aligned}
$$

Similarly, we propose an estimator using pth raw moments, given by

$$
\widehat{\bar{Y}}_{P}=\bar{y}^{*}\left(\frac{C \bar{u}^{*}+D}{C \bar{u}^{\prime}+D}\right)^{\gamma}\left(\frac{C \bar{u}+D}{A \bar{u}^{\prime}+D}\right)^{\delta},
$$

where $\bar{u}=\sum_{i=1}^{n} x_{i}^{p} / n$ and $\bar{u}^{\prime}=\sum_{i=1}^{n^{\prime}} x_{i}^{p} / n^{\prime} ; \gamma$ and $\delta$ are constants; $C$ and $D$ are known population parameters.

Replacing $\bar{y}^{*}$ in (9) by $\widehat{\bar{Y}}_{P}$ from (12), we suggest the following estimator:

$$
\begin{aligned}
\widehat{\bar{Y}}_{M}= & \bar{y}^{*}\left(\frac{A \bar{x}^{*}+B}{A \bar{x}^{\prime}+B}\right)^{\alpha}\left(\frac{A \bar{x}+B}{A \bar{x}^{\prime}+B}\right)^{\beta} \\
& \times\left(\frac{C \bar{u}^{*}+D}{C \bar{u}^{\prime}+D}\right)^{\gamma}\left(\frac{C \bar{u}+D}{A \bar{u}^{\prime}+D}\right)^{\delta} .
\end{aligned}
$$

Now expressing $\widehat{\bar{Y}}_{M}$ in terms of $e$ 's, we have

$$
\begin{aligned}
\hat{\bar{Y}}_{M}= & \left(1+e_{0}^{*}\right) \bar{Y}\left(\frac{1+\phi e_{1}^{*}}{1+\phi e_{1}^{\prime}}\right)^{\alpha}\left(\frac{1+\phi e_{1}}{1+\phi e_{1}^{\prime}}\right)^{\beta} \\
& \times\left(\frac{1+\psi e_{2}^{*}}{1+\psi e_{2}^{\prime}}\right)^{\gamma}\left(\frac{1+\psi e_{2}}{1+\psi e_{2}^{\prime}}\right)^{\delta}
\end{aligned}
$$

where $\psi=(C \bar{U} /(C \bar{U}+D))$. 
We assume that $\left|\phi e_{i}^{\prime}\right|<1\left|\phi e_{i}\right|<1\left|\phi e_{i}^{*}\right|<1$ for $i=1,2$, such that the right hand side of (14) is expandable. Solving (14), we have

$$
\begin{aligned}
& \left(\widehat{\bar{Y}}_{M}-\bar{Y}\right) \\
& \cong \bar{Y}\left[e_{0}^{*}+\alpha \phi\left(e_{1}^{*}+e_{0}^{*} e_{1}^{*}-e_{0}^{*} e_{1}^{\prime}\right)+\beta \phi\left(e_{1}+e_{0}^{*} e_{1}-e_{0}^{*} e_{1}^{\prime}\right)\right. \\
& +\gamma \psi\left(e_{2}^{*}+e_{0}^{*} e_{2}^{*}-e_{0}^{*} e_{2}^{\prime}\right)+\delta \psi\left(e_{2}+e_{0}^{*} e_{2}-e_{0}^{*} e_{2}^{\prime}\right) \\
& -(\alpha+\beta) \phi e_{1}^{\prime}-(\gamma+\delta) \psi e_{2}^{\prime} \\
& +\alpha \beta \phi^{2}\left(e_{1}^{\prime 2}-e_{1} e_{1}^{\prime}+e_{1}^{*} e_{1}+e_{1}^{*} e_{1}^{\prime}\right) \\
& +\gamma \delta \psi^{2}\left(e_{2}^{\prime 2}-e_{2} e_{2}^{\prime}+e_{2}^{*} e_{2}+e_{2}^{*} e_{2}^{\prime}\right) \\
& +\alpha \beta \phi^{2}\left(e_{1}^{\prime 2}-e_{1} e_{1}^{\prime}+e_{1}^{*} e_{1}+e_{1}^{*} e_{1}^{\prime}\right) \\
& +\gamma \delta \psi^{2}\left(e_{2}^{\prime 2}-e_{2} e_{2}^{\prime}+e_{2}^{*} e_{2}+e_{2}^{*} e_{2}^{\prime}\right) \\
& +\alpha \beta \phi^{2}\left(e_{1}^{\prime 2}-e_{1} e_{1}^{\prime}+e_{1}^{*} e_{1}+e_{1}^{*} e_{1}^{\prime}\right) \\
& +\gamma \delta \psi^{2}\left(e_{2}^{\prime 2}-e_{2} e_{2}^{\prime}+e_{2}^{*} e_{2}+e_{2}^{*} e_{2}^{\prime}\right) \\
& +\frac{\psi^{2}}{2} \beta\left\{(\beta+1) e_{1}^{\prime 2}+(\beta-1) e_{1}^{2}\right\} \\
& +\frac{\psi^{2}}{2} \gamma\left\{(\gamma+1) e_{2}^{\prime 2}+(\gamma-1) e_{2}^{* 2}\right\} \\
& +\frac{\psi^{2}}{2} \delta\left\{(\delta+1) e_{2}^{\prime 2}+(\delta-1) e_{2}^{2}\right\} \\
& +\alpha \gamma \phi \psi\left(e_{1}^{*} e_{2}^{*}-e_{1}^{\prime} e_{2}^{*}-e_{2}^{\prime} e_{1}^{*}+e_{1}^{\prime} e_{2}^{\prime}\right) \\
& +\beta \gamma \phi \psi\left(e_{1}^{\prime} e_{2}^{*}-e_{1}^{\prime} e_{2}^{*}-e_{1} e_{2}^{\prime}+e_{1}^{\prime} e_{2}^{\prime}\right) \\
& +\alpha \delta \phi \psi\left(e_{1}^{*} e_{2}-e_{1}^{\prime} e_{2}-e_{2}^{\prime} e_{1}^{*}+e_{1}^{\prime} e_{2}^{\prime}\right) \\
& \left.+\beta \delta \phi \psi\left(e_{1} e_{2}-e_{1}^{\prime} e_{2}-e_{2}^{\prime} e_{1}+e_{1}^{\prime} e_{2}^{\prime}\right)\right] \text {. }
\end{aligned}
$$

Taking expectation of (15), the bias of $\widehat{\bar{Y}}_{M}$ to the first degree of approximation is given by

$$
\begin{gathered}
B\left(\widehat{\bar{Y}}_{M}\right) \\
\cong \bar{Y}\left[\left\{( \lambda - \lambda ^ { \prime } ) \phi \left(\alpha\left(k_{y x}+\frac{\alpha-1}{2} \phi\right)\right.\right.\right. \\
\left.+\beta\left(k_{y x}+\alpha \phi+\frac{\beta-1}{2} \phi\right)\right) C_{x}^{2} \\
+\psi\left(\gamma\left(k_{y u}+\frac{\gamma-1}{2} \psi\right)+\delta\left(k_{y u}+\gamma \psi+\frac{\delta-1}{2} \psi\right)\right. \\
\left.+\phi(\alpha \gamma+\beta \gamma+\alpha \delta+\beta \delta)) k_{x u} C_{u}^{2}\right\} \\
+\lambda^{*}\left(\alpha \phi\left(k_{y x(2)}+\frac{\alpha-1}{2} \phi\right) C_{x(2)}^{2}\right. \\
\left.\left.+\gamma \psi\left(k_{y u(2)}+\frac{\gamma-1}{2} \psi+\alpha \phi k_{x u(2)}\right) C_{u(2)}^{2}\right)\right]
\end{gathered}
$$

Squaring both sides of (15) and neglecting e's involving power greater than two and then taking expectation, we get mean square error of the suggested class of estimators to the first degree of approximation as

$$
\begin{aligned}
& M\left(\hat{\bar{Y}}_{M}\right) \\
& \cong \bar{Y}^{2}\left[\left(\lambda-\lambda^{\prime}\right)\right. \\
& \times\left\{C_{y}^{2}+(\alpha+\beta) \phi\left((\alpha+\beta) \phi+2 k_{y x}\right) C_{x}^{2}\right. \\
& +(\gamma+\delta) \psi\left((\gamma+\delta) \psi+2 k_{y u}\right) C_{u}^{2} \\
& \left.+2(\alpha+\beta)(\gamma+\delta) \phi \psi k_{x u} C_{u}^{2}\right\} \\
& +\lambda^{*}\left\{C_{y(2)}^{2}+\alpha \phi\left(\alpha \phi+2 k_{y x(2)}\right) C_{x(2)}^{2}\right. \\
& +\gamma \psi\left(\gamma \psi+2 k_{y u(2)}\right) C_{u(2)}^{2} \\
& \left.\left.+2 \alpha \gamma \phi \psi k_{x u(2)} C_{u(2)}^{2}\right\}+\lambda^{\prime} C_{y}^{2}\right] .
\end{aligned}
$$

From (17) the optimum values of $\alpha, \beta, \gamma$, and $\delta$ are given by

$$
\begin{gathered}
\alpha=-\left\{\frac{k_{y x(2)}-k_{y u(2)} k_{u x(2)}}{\phi\left(1-\rho_{x u(2)}^{2}\right)}\right\}, \\
\beta=-\left\{\frac{k_{y x}-k_{y u} k_{u x}}{\phi\left(1-\rho_{x u}^{2}\right)}\right\}+\left\{\frac{k_{y x(2)}-k_{y u(2)} k_{u x(2)}}{\phi\left(1-\rho_{x u(2)}^{2}\right)}\right\}, \\
\gamma=-\left\{\frac{k_{y u(2)}-k_{y x(2)} k_{x u(2)}}{\psi\left(1-\rho_{x u(2)}^{2}\right)}\right\}, \\
\delta=-\left\{\frac{k_{y u}-k_{y x} k_{x u}}{\psi\left(1-\rho_{x u}^{2}\right)}\right\}+\left\{\frac{k_{y u(2)}-k_{y x(2)} k_{x u(2)}}{\psi\left(1-\rho_{x u(2)}^{2}\right)}\right\} .
\end{gathered}
$$

Substituting the optimum values of $\alpha, \beta, \gamma$, and $\delta$ in (17) and using the identity

$$
\begin{gathered}
\rho_{x u(2)}^{2}=k_{u x(2)} k_{x u(2)}, \\
\rho_{x u}^{2}=k_{u x} k_{x u}, \quad \rho_{y u(2)}^{2}=k_{y u(2)} k_{u y(2)}, \\
\rho_{y u}^{2}=k_{y u} k_{u y}, \quad \rho_{y x(2)}^{2}=k_{y x(2)} k_{x y(2)}, \\
\rho_{y x}^{2}=k_{y x} k_{x y},
\end{gathered}
$$


we get the optimum mean square error of $\widehat{\bar{Y}}_{M}$, given by

$$
\begin{aligned}
& M\left(\hat{\bar{Y}}_{M}\right)_{\mathrm{opt}} \\
& \cong \bar{Y}^{2}\left[\left(\lambda-\lambda^{\prime}\right) C_{y}^{2}\left\{1-\frac{\left(\rho_{y x}^{2}+\rho_{y u}^{2}-2 \rho_{y x} \rho_{y u} \rho_{x u}\right)}{\left(1-\rho_{x u}^{2}\right)}\right\}\right. \\
& +\lambda^{*} C_{y}^{2}\left\{1-\frac{\left(\rho_{y x(2)}^{2}+\rho_{y u(2)}^{2}-2 \rho_{y x(2)} \rho_{y u(2)} \rho_{x u(2)}\right)}{\left(1-\rho_{x u(2)}^{2}\right)}\right\} \\
& \left.+\lambda^{\prime} C_{y}^{2}\right] .
\end{aligned}
$$

Similarly the bias and mean square error of $\widehat{\bar{Y}}_{P}$ given in (12) can be deduced from (16) and (17) by putting $\alpha=\beta=0$, given by

$$
\begin{gathered}
B\left(\hat{\bar{Y}}_{P}\right) \\
\cong \bar{Y}\left[( \lambda - \lambda ^ { \prime } ) \psi \left(\gamma\left(k_{y u}+\frac{\gamma-1}{2} \psi\right)\right.\right. \\
\left.+\delta\left(k_{y u}+\gamma \psi+\frac{\delta-1}{2} \psi\right)\right) C_{u}^{2} \\
\left.+\lambda^{*}\left(\gamma \psi\left(k_{y u(2)}+\frac{\gamma-1}{2} \psi\right) C_{u(2)}^{2}\right)\right], \\
M\left(\widehat{\bar{Y}}_{P}\right)+\bar{Y}^{2}\left[\left(\lambda-\lambda^{\prime}\right)\left\{C_{y}^{2}+(\gamma+\delta) \psi\left((\gamma+\delta) \psi+2 k_{y u}\right) C_{u}^{2}\right\}\right. \\
\left.+\lambda^{*}\left\{C_{y(2)}^{2}+\gamma \psi\left(\gamma \psi+2 k_{y u(2)}\right) C_{u(2)}^{2}\right\}+\lambda^{\prime} C_{y}^{2}\right] .
\end{gathered}
$$

The mean square error of $\widehat{\bar{Y}}_{P}$ for optimum values of $\gamma$ and $\delta$ is given by

$$
\begin{aligned}
M\left(\hat{\bar{Y}}_{P}\right)_{\mathrm{opt}} \cong \bar{Y}^{2}[ & \left(\lambda-\lambda^{\prime}\right) C_{y}^{2}\left\{1-\rho_{y u}^{2}\right\} \\
& \left.+\lambda^{*} C_{y}^{2}\left\{1-\rho_{y u(2)}^{2}\right\}+\lambda^{\prime} C_{y}^{2}\right] .
\end{aligned}
$$

\section{Some Members of the Proposed Family of Estimators $\widehat{\bar{Y}}_{M}$}

In Table 1, we give some members of a proposed family of estimators.

For $p=1$, the estimators given in Table 1 reduce to usual ratio and product type estimators.

The biases and mean square errors of the estimators $\widehat{\bar{Y}}_{M i}$ for $i=1,2, \ldots, 10$, to the first degree of approximation are given in Table 2 .
TABLE 1: Some members of a proposed family of estimators for $\alpha=$ $\beta=0$ in (13).

\begin{tabular}{lllll}
\hline Estimator & $\gamma$ & $\delta$ & $C$ & $D$ \\
$\hat{\bar{Y}}_{M 0}=\bar{y}^{*}$ & 0 & 0 & - & - \\
$\hat{\bar{Y}}_{M 1}=\bar{y}^{*}\left(\frac{\bar{u}^{\prime}}{\bar{u}^{*}}\right)$ & -1 & 0 & 1 & 0 \\
$\hat{\bar{Y}}_{M 2}=\bar{y}^{*}\left(\frac{\bar{u}^{\prime}}{\bar{u}}\right)$ & 0 & -1 & 1 & 0 \\
$\hat{\bar{Y}}_{M 3}=\bar{y}^{*}\left(\frac{\bar{u}^{\prime}}{\bar{u}^{*}}\right)\left(\frac{\bar{u}^{\prime}}{\bar{u}}\right)$ & -1 & -1 & 1 & 0 \\
$\hat{\bar{Y}}_{M 4}=\bar{y}^{*}\left(\frac{\bar{u}^{*}}{\bar{u}^{\prime}}\right)\left(\frac{\bar{u}^{\prime}}{\bar{u}}\right)$ & 1 & 1 & 1 & 0 \\
$\hat{\bar{Y}}_{M 5}=\bar{y}^{*}\left(\frac{\bar{u}^{*}+C_{u}}{\bar{u}^{\prime}+C_{u}}\right)$ & -1 & 0 & 1 & $C_{u}$ \\
$\hat{\bar{Y}}_{M 6}=\bar{y}^{*}\left(\frac{\bar{u}^{\prime}+C_{u}}{\bar{u}^{\prime}+C_{u}}\right)$ & -1 & 1 & $C_{u}$ \\
$\hat{\bar{Y}}_{M 7}=\bar{y}^{*}\left(\frac{\bar{u}^{\prime}+C_{u}}{\bar{u}^{*}+C_{u}}\right)\left(\frac{\bar{u}^{\prime}+C_{u}}{\bar{u}+C_{u}}\right)$ & -1 & -1 & 1 & $C_{u}$ \\
$\hat{\bar{Y}}_{M 8}=\bar{y}^{*}\left(\frac{\bar{u}^{\prime}+\rho_{y u}}{\bar{u}^{*}+\rho_{y u}}\right)$ & -1 & 0 & 1 & $\rho_{y u}$ \\
$\hat{\bar{Y}}_{M 9}=\bar{y}^{*}\left(\frac{\bar{u}^{\prime}+\rho_{y u}}{\bar{u}^{\prime}+\rho_{y u}}\right)$ & & & & \\
$\hat{\bar{Y}}_{M 10}=\bar{y}^{*}\left(\frac{\bar{u}^{\prime}+\rho_{y u}}{\bar{u}^{*}+\rho_{y u}}\right)\left(\frac{\bar{u}^{\prime}+\rho_{y u}}{\bar{u}+\rho_{y u}}\right)$ & -1 & 1 & $\rho_{y u}$ \\
\hline
\end{tabular}

\section{Efficiency Comparison}

A proposed class of estimators $\widehat{\bar{Y}}_{M}$ will perform better than the following:

(i) usual unbiased estimator $\widehat{\bar{Y}}_{M 0}$ if

$$
\begin{aligned}
& \left\{0>\gamma>\max \left\{-\left(\delta+\frac{2 k_{y u}}{\psi}\right) ;-\frac{2 k_{y u(2)}}{\psi}\right\}\right. \\
& \text { or } \left.0<\gamma<\min \left\{-\left(\delta+\frac{2 k_{y u}}{\psi}\right) ;-\frac{2 k_{y u(2)}}{\psi}\right\}\right\} \text {, } \\
& 0>\alpha>\max \left\{-\left(\beta+\frac{\left(2 k_{y x}+2(\gamma+\delta) k_{u x}\right)}{\phi}\right)\right. \text {; } \\
& \left.-\frac{\left(2 k_{y x(2)}+2 \gamma k_{u x(2)}\right)}{\phi}\right\} \\
& \text { or } 0<\alpha<\min \left\{-\left(\beta+\frac{\left(2 k_{y x}+2(\gamma+\delta) k_{u x}\right)}{\phi}\right)\right. \text {; } \\
& \left.-\frac{\left(2 k_{y x(2)}+2 \gamma k_{u x(2)}\right)}{\phi}\right\}
\end{aligned}
$$




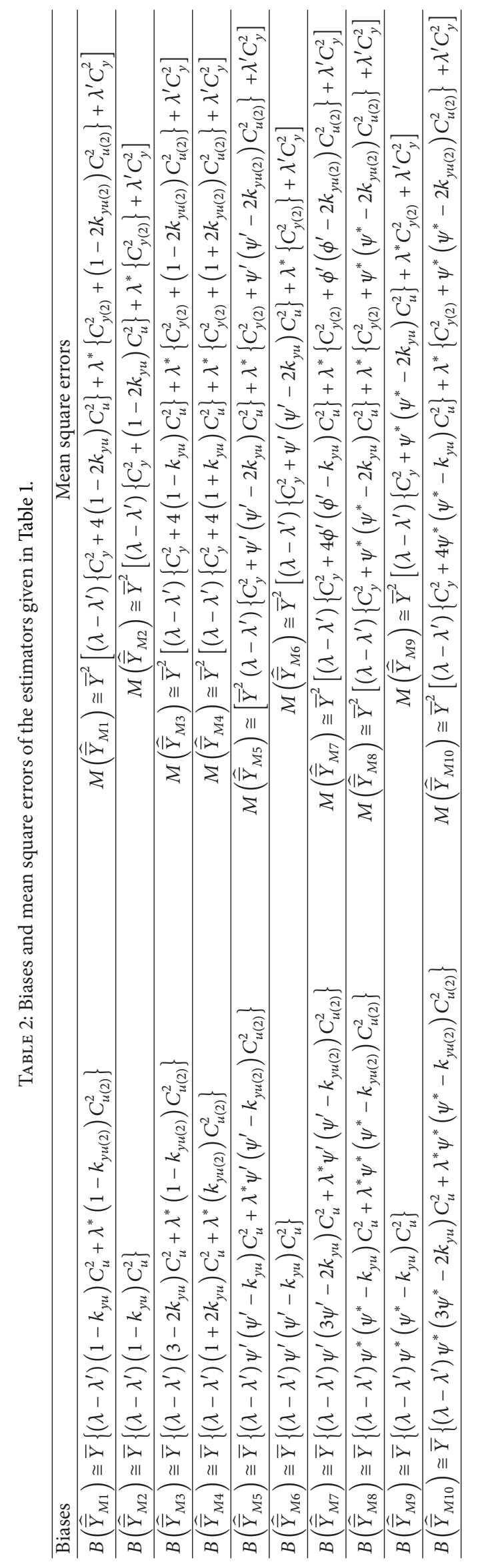


(ii) the usual ratio estimator $\hat{\bar{Y}}_{M 1}$ if

$$
\begin{aligned}
0>\gamma>\max & \left\{-\left(\delta+\frac{\left(2 k_{y u}-1\right)}{\psi}\right) ;-\frac{\left(2 k_{y u(2)}-1\right)}{\psi}\right\} \\
\text { or } 0<\gamma<\min \{- & \left.\left(\delta+\frac{\left(2 k_{y u}-1\right)}{\psi}\right) ;-\frac{\left(2 k_{y u(2)}-1\right)}{\psi}\right\}, \\
0>\alpha>\max \{- & \left(\beta+\frac{\left(2 k_{y x}+2(\gamma+\delta) k_{u x}\right)}{\phi}\right) ; \\
& \left.-\frac{\left(2 k_{y x(2)}+2 \gamma k_{u x(2)}\right)}{\phi}\right\} \\
\text { or } 0<\alpha<\min \{ & -\left(\beta+\frac{\left(2 k_{y x}+2(\gamma+\delta) k_{u x}\right)}{\phi}\right) ; \\
& \left.-\frac{\left(2 k_{y x(2)}+2 \gamma k_{u x(2)}\right)}{\phi}\right\} ;
\end{aligned}
$$

(iii) the ratio estimator $\widehat{\bar{Y}}_{M 2}$ if

$$
\begin{aligned}
0>\gamma>\max & \left\{-\left(\delta+\frac{\left(2 k_{y u}-1\right)}{\psi}\right) ;-\frac{\left.2 k_{y u(2)}\right\}}{\psi}\right\} \\
\text { or } 0<\gamma<\min \{ & \left.-\left(\delta+\frac{\left(2 k_{y u}-1\right)}{\psi}\right) ;-\frac{2 k_{y u(2)}}{\psi}\right\}, \\
0>\alpha>\max \{ & -\left(\beta+\frac{\left(2 k_{y x}+2(\gamma+\delta) k_{u x}\right)}{\phi}\right) ; \\
& \left.-\frac{\left(2 k_{y x(2)}+2 \gamma k_{u x(2)}\right)}{\phi}\right\} \\
\text { or } 0<\alpha<\min \{ & \left\{-\left(\beta+\frac{\left(2 k_{y x}+2(\gamma+\delta) k_{u x}\right)}{\phi}\right) ;\right. \\
& \left.-\frac{\left(2 k_{y x(2)}+2 \gamma k_{u x(2)}\right)}{\phi}\right\} ;
\end{aligned}
$$

(iv) the ratio estimator $\widehat{\bar{Y}}_{M 3}$ if

$$
\begin{aligned}
0>\gamma>\max & \left\{-\left(\delta+\frac{2\left(k_{y u}-1\right)}{\psi}\right) ;-\frac{\left(2 k_{y u(2)}-1\right)}{\psi}\right\} \\
\text { or } 0<\gamma<\min \{- & \left.\left(\delta+\frac{2\left(k_{y u}-1\right)}{\psi}\right) ;-\frac{\left(2 k_{y u(2)}-1\right)}{\psi}\right\}, \\
0>\alpha>\max \{- & \left(\beta+\frac{\left(2 k_{y x}+2(\gamma+\delta) k_{u x}\right)}{\phi}\right) ; \\
& \left.-\frac{\left(2 k_{y x(2)}+2 \gamma k_{u x(2)}\right)}{\phi}\right\}
\end{aligned}
$$

$$
\text { or } 0<\alpha<\min \left\{\begin{aligned}
- & \left(\beta+\frac{\left(2 k_{y x}+2(\gamma+\delta) k_{u x}\right)}{\phi}\right) ; \\
& \left.-\frac{\left(2 k_{y x(2)}+2 \gamma k_{u x(2)}\right)}{\phi}\right\} ;
\end{aligned}\right.
$$

(v) the ratio estimator $\hat{\bar{Y}}_{M 4}$ if

$$
\begin{aligned}
0>\gamma>\max \{- & \left.\left(\delta+\frac{2\left(k_{y u}+1\right)}{\psi}\right) ;-\frac{\left(2 k_{y u(2)}+1\right)}{\psi}\right\} \\
\text { or } 0<\gamma<\min \{- & \left.\left(\delta+\frac{2\left(k_{y u}+1\right)}{\psi}\right) ;-\frac{\left(2 k_{y u(2)}+1\right)}{\psi}\right\}, \\
0>\alpha>\max \{- & \left(\beta+\frac{\left(2 k_{y x}+2(\gamma+\delta) k_{u x}\right)}{\phi}\right) ; \\
& \left.-\frac{\left(2 k_{y x(2)}+2 \gamma k_{u x(2)}\right)}{\phi}\right\} \\
\text { or } 0<\alpha<\min \{- & \left(\beta+\frac{\left(2 k_{y x}+2(\gamma+\delta) k_{u x}\right)}{\phi}\right) ; \\
& \left.-\frac{\left(2 k_{y x(2)}+2 \gamma k_{u x(2)}\right)}{\phi}\right\} ;
\end{aligned}
$$

(vi) the ratio estimator $\hat{\bar{Y}}_{M 5}$ if

$$
\begin{gathered}
0>\gamma>\max \left\{-\left(\delta+\frac{\left(2 k_{y u}-\psi^{\prime}\right)}{\psi}\right) ;-\frac{\left(2 k_{y u(2)}-\psi^{\prime}\right)}{\psi}\right\} \\
\text { or } 0<\gamma<\min \left\{-\left(\delta+\frac{\left(2 k_{y u}-\psi^{\prime}\right)}{\psi}\right) ;-\frac{\left(2 k_{y u(2)}-\psi^{\prime}\right)}{\psi}\right\},
\end{gathered}
$$$$
0>\alpha>\max \left\{-\left(\beta+\frac{\left(2 k_{y x}+2(\gamma+\delta) k_{u x}\right)}{\phi}\right)\right. \text {; }
$$

$$
\left.-\frac{\left(2 k_{y x(2)}+2 \gamma k_{u x(2)}\right)}{\phi}\right\}
$$$$
\text { or } 0<\alpha<\min \left\{-\left(\beta+\frac{\left(2 k_{y x}+2(\gamma+\delta) k_{u x}\right)}{\phi}\right)\right. \text {; }
$$

$$
\left.-\frac{\left(2 k_{y x(2)}+2 \gamma k_{u x(2)}\right)}{\phi}\right\} ;
$$


(vii) the ratio estimator $\widehat{\bar{Y}}_{M 6}$ if

$$
\begin{aligned}
& 0>\gamma>\max \left\{-\left(\delta+\frac{\left(2 k_{y u}-\psi^{\prime}\right)}{\psi}\right) ;-\frac{2 k_{y u(2)}}{\psi}\right\} \\
& \text { or } 0<\gamma<\min \left\{-\left(\delta+\frac{\left(2 k_{y u}-\psi^{\prime}\right)}{\psi}\right) ;-\frac{2 k_{y u(2)}}{\psi}\right\} \text {, } \\
& 0>\alpha>\max \left\{-\left(\beta+\frac{\left(2 k_{y x}+2(\gamma+\delta) k_{u x}\right)}{\phi}\right)\right. \text {; } \\
& \left.-\frac{\left(2 k_{y x(2)}+2 \gamma k_{u x(2)}\right)}{\phi}\right\} \\
& \text { or } 0<\alpha<\min \left\{-\left(\beta+\frac{\left(2 k_{y x}+2(\gamma+\delta) k_{u x}\right)}{\phi}\right)\right. \text {; } \\
& \left.-\frac{\left(2 k_{y x(2)}+2 \gamma k_{u x(2)}\right)}{\phi}\right\} \text {; }
\end{aligned}
$$

(viii) the ratio estimator $\widehat{\bar{Y}}_{M 7}$ if

$$
\begin{aligned}
0>\gamma>\max & \left\{-\left(\delta+\frac{\left(2 k_{y u}-\psi^{\prime}\right)}{\psi}\right) ;-\frac{2 k_{y u(2)}}{\psi}\right\} \\
\text { or } 0<\gamma<\min \{- & \left.\left(\delta+\frac{\left(2 k_{y u}-\psi^{\prime}\right)}{\psi}\right) ;-\frac{2 k_{y u(2)}}{\psi}\right\}, \\
0>\alpha>\max \{- & \left(\beta+\frac{\left(2 k_{y x}+2(\gamma+\delta) k_{u x}\right)}{\phi}\right) ; \\
& \left.-\frac{\left(2 k_{y x(2)}+2 \gamma k_{u x(2)}\right)}{\phi}\right\}
\end{aligned}
$$$$
\text { or } 0<\alpha<\min \left\{-\left(\beta+\frac{\left(2 k_{y x}+2(\gamma+\delta) k_{u x}\right)}{\phi}\right)\right. \text {; }
$$$$
\left.-\frac{\left(2 k_{y x(2)}+2 \gamma k_{u x(2)}\right)}{\phi}\right\} \text {; }
$$

(ix) the ratio estimator $\widehat{\bar{Y}}_{M 8}$ if

$$
\begin{gathered}
0>\gamma>\max \left\{-\left(\delta+\frac{\left(2 k_{y u}-\psi^{*}\right)}{\psi}\right) ;-\frac{\left(2 k_{y u(2)}-\psi^{*}\right)}{\psi}\right\}, \\
\text { or } 0<\gamma<\min \left\{-\left(\delta+\frac{\left(2 k_{y u}-\psi^{*}\right)}{\psi}\right) ;-\frac{\left(2 k_{y u(2)}-\psi^{*}\right)}{\psi}\right\}, \\
0>\alpha>\max \left\{-\left(\beta+\frac{\left(2 k_{y x}+2(\gamma+\delta) k_{u x}\right)}{\phi}\right) ;\right. \\
\left.-\frac{\left(2 k_{y x(2)}+2 \gamma k_{u x(2)}\right)}{\phi}\right\}
\end{gathered}
$$

$$
\begin{aligned}
& \text { or } 0<\alpha<\min \left\{-\left(\beta+\frac{\left(2 k_{y x}+2(\gamma+\delta) k_{u x}\right)}{\phi}\right)\right. \text {; } \\
& \left.-\frac{\left(2 k_{y x(2)}+2 \gamma k_{u x(2)}\right)}{\phi}\right\} \text {; }
\end{aligned}
$$

(x) the ratio estimator $\hat{\bar{Y}}_{M 9}$ if

$$
\begin{aligned}
& 0>\gamma>\max \left\{-\left(\delta+\frac{\left(2 k_{y u}-\psi^{*}\right)}{\psi}\right) ;-\frac{2 k_{y u(2)}}{\psi}\right\} \\
& \text { or } 0<\gamma<\min \left\{-\left(\delta+\frac{\left(2 k_{y u}-\psi^{*}\right)}{\psi}\right) ;-\frac{2 k_{y u(2)}}{\psi}\right\} \text {, } \\
& 0>\alpha>\max \left\{-\left(\beta+\frac{\left(2 k_{y x}+2(\gamma+\delta) k_{u x}\right)}{\phi}\right)\right. \text {; } \\
& \left.-\frac{\left(2 k_{y x(2)}+2 \gamma k_{u x(2)}\right)}{\phi}\right\} \\
& \text { or } 0<\alpha<\min \left\{-\left(\beta+\frac{\left(2 k_{y x}+2(\gamma+\delta) k_{u x}\right)}{\phi}\right)\right. \text {; } \\
& \left.-\frac{\left(2 k_{y x(2)}+2 \gamma k_{u x(2)}\right)}{\phi}\right\}
\end{aligned}
$$

(xi) the ratio estimator $\hat{\bar{Y}}_{M 10}$ if

$$
\begin{gathered}
0>\gamma>\max \left\{-\left(\delta+\frac{2\left(k_{y u}-\psi^{*}\right)}{\psi}\right) ;-\frac{\left(2 k_{y u(2)}-\psi^{*}\right)}{\psi}\right\} \\
\text { or } 0<\gamma<\min \left\{-\left(\delta+\frac{2\left(k_{y u}-\psi^{*}\right)}{\psi}\right) ;-\frac{\left(2 k_{y u(2)}-\psi^{*}\right)}{\psi}\right\},
\end{gathered}
$$$$
0>\alpha>\max \left\{-\left(\beta+\frac{\left(2 k_{y x}+2(\gamma+\delta) k_{u x}\right)}{\phi}\right)\right. \text {; }
$$

$$
\left.-\frac{\left(2 k_{y x(2)}+2 \gamma k_{u x(2)}\right)}{\phi}\right\}
$$$$
\text { or } 0<\alpha<\min \left\{-\left(\beta+\frac{\left(2 k_{y x}+2(\gamma+\delta) k_{u x}\right)}{\phi}\right)\right. \text {; }
$$

$$
\left.-\frac{\left(2 k_{y x(2)}+2 \gamma k_{u x(2)}\right)}{\phi}\right\}
$$


TABLE 3: Percent relative efficiency of different estimators with respect to $\bar{y}^{*}$.

\begin{tabular}{|c|c|c|c|c|c|c|}
\hline \multirow{2}{*}{ Estimator } & \multicolumn{3}{|c|}{ For $p=1$ in (13) } & \multicolumn{3}{|c|}{ For $p=0.8$ in (13) } \\
\hline & $(k, r)=(2,6)$ & $(k, r)=(3,4)$ & $(k, r)=(4,3)$ & $(k, r)=(2,6)$ & $(k, r)=(3,4)$ & $(k, r)=(4,3)$ \\
\hline $\bar{y}^{*}$ & 100.00 & 100.00 & 100.00 & 100.00 & 100.00 & 100.00 \\
\hline$\hat{\bar{Y}}_{M 1}$ & 29.09 & 31.94 & 34.65 & 54.02 & 57.72 & 61.06 \\
\hline$\hat{\bar{Y}}_{M 2}$ & 74.56 & 68.74 & 64.70 & 91.12 & 88.51 & 86.52 \\
\hline$\overline{\bar{Y}}_{M 3}$ & 16.85 & 15.48 & 14.54 & 29.75 & 26.69 & 24.67 \\
\hline$\overline{\bar{Y}}_{M 4}$ & 06.26 & 06.23 & 06.20 & 8.62 & 8.60 & 8.58 \\
\hline$\widehat{\bar{Y}}_{M 5}$ & 29.35 & 32.22 & 34.94 & 55.38 & 59.09 & 62.42 \\
\hline$\widehat{\bar{Y}}_{M 6}$ & 74.82 & 69.04 & 65.01 & 91.71 & 89.25 & 87.37 \\
\hline$\overline{\bar{Y}}_{M 7}$ & 16.99 & 15.61 & 14.65 & 30.45 & 27.30 & 25.21 \\
\hline$\overline{\bar{Y}}_{M 8}$ & 29.34 & 32.21 & 34.93 & 55.68 & 59.39 & 62.72 \\
\hline$\widehat{\overline{\bar{Y}}}_{M 9}$ & 74.81 & 69.03 & 65.01 & 91.83 & 89.41 & 87.55 \\
\hline$\widehat{\bar{Y}}_{M 10}$ & 16.99 & 15.60 & 14.65 & 30.60 & 27.30 & 25.33 \\
\hline$\hat{\overline{\bar{Y}}}_{\mathrm{SK}}$ & 314.68 & 258.16 & 225.75 & 314.68 & 258.16 & 225.75 \\
\hline$\hat{\bar{Y}}_{P}$ & 314.68 & 258.16 & 225.75 & 339.39 & 273.28 & 236.45 \\
\hline$\widehat{\bar{Y}}_{M}$ & 314.68 & 258.16 & 225.75 & 398.61 & 307.40 & 259.86 \\
\hline
\end{tabular}

TABLE 4: Percent relative efficiency of different estimator with respect to $\bar{y}^{*}$.

\begin{tabular}{|c|c|c|c|c|c|c|}
\hline \multirow{2}{*}{ Estimator } & \multicolumn{3}{|c|}{ For $p=0.5$ in (13) } & \multicolumn{3}{|c|}{ For $p=0.35$ in (13) } \\
\hline & $(k, r)=(2,6)$ & $(k, r)=(3,4)$ & $(k, r)=(4,3)$ & $(k, r)=(2,6)$ & $(k, r)=(3,4)$ & $(k, r)=(4,3)$ \\
\hline $\bar{y}^{*}$ & 100.00 & 100.00 & 100.00 & 100.00 & 100.00 & 100.00 \\
\hline$\overline{\bar{Y}}_{M 1}$ & 220.99 & 199.59 & 185.25 & 394.52 & 305.13 & 258.33 \\
\hline$\overline{\bar{Y}}_{M 2}$ & 112.95 & 118.04 & 122.45 & 116.43 & 123.17 & 129.15 \\
\hline$\overline{\bar{Y}}_{M 3}$ & 112.15 & 92.05 & 80.51 & 263.47 & 201.70 & 169.86 \\
\hline$\widehat{\widehat{\bar{Y}}}_{M 4}$ & 16.38 & 16.42 & 16.45 & 24.95 & 25.10 & 25.23 \\
\hline$\widehat{\bar{Y}}_{M 5}$ & 238.20 & 211.70 & 194.40 & 395.11 & 305.07 & 258.06 \\
\hline$\overline{\bar{Y}}_{M 6}$ & 113.55 & 118.92 & 123.58 & 116.34 & 123.03 & 128.97 \\
\hline$\overline{\bar{Y}}_{M 7}$ & 121.82 & 99.31 & 86.53 & 281.96 & 215.91 & 181.85 \\
\hline$\widehat{\bar{Y}}_{M 8}$ & 255.10 & 223.20 & 202.91 & 383.69 & 298.38 & 253.34 \\
\hline $\bar{Y}_{M 9}$ & 114.06 & 119.66 & 124.55 & 116.00 & 122.53 & 128.31 \\
\hline$\widehat{\bar{Y}}_{M 10}$ & 131.79 & 106.73 & 92.65 & 307.09 & 237.08 & 200.53 \\
\hline$\hat{\bar{Y}}_{\mathrm{SK}}$ & 314.68 & 258.16 & 225.75 & 314.68 & 258.16 & 225.75 \\
\hline$\overline{\bar{Y}}_{P}$ & 378.35 & 295.98 & 252.11 & 395.97 & 305.87 & 258.79 \\
\hline$\overline{\bar{Y}}_{M}$ & 407.31 & 312.26 & 263.16 & 411.30 & 314.48 & 264.67 \\
\hline
\end{tabular}

(xii) the Singh and Kumar [13] estimator $\hat{\bar{Y}}_{\mathrm{SK}}$ if

$$
\begin{aligned}
& 0>\alpha>\max \{-\left(\beta+\frac{\left(2 k_{y x}+2(\gamma+\delta) k_{u x}\right)}{\phi}\right) ; \\
& \text { or } 0<\alpha<\min \{--\left(\beta+\frac{\left(2 k_{y x(2)}+2 \gamma k_{u x(2)}\right)}{\phi}\right\} \\
&\left.-\frac{\left(2 k_{y x}+2(\gamma+\delta) k_{u x}\right)}{\phi}\right) ; \\
& \phi
\end{aligned}
$$

4.1. Comparison of Proposed Estimator with the Singh and Kumar [13] Estimator. Comparison of a proposed estimator $\widehat{\bar{Y}}_{P}$ with other estimators given in Table 1 will yield similar expressions already derived by Singh and Kumar [13].

(i) For optimum values of the constant involved $\hat{\bar{Y}}_{M}$ will be more efficient than $\hat{\bar{Y}}_{\mathrm{SK}}$; that is,

$$
M\left(\hat{\bar{Y}}_{M}\right)_{\mathrm{opt}}<M\left(\hat{\bar{Y}}_{\mathrm{SK}}\right)_{\mathrm{opt}}
$$


TABle 5: Percent relative efficiency of different estimator with respect to $\bar{y}^{*}$ for $p=0.3$ in (13).

\begin{tabular}{lccc}
\hline Estimator & $(k, r)=(2,6)$ & $(k, r)=(3,4)$ & $(k, r)=(4,3)$ \\
\hline $\bar{y}^{*}$ & 100.00 & 100.00 & 100.00 \\
$\widehat{\bar{Y}}_{M 1}$ & 382.76 & 297.88 & 253.02 \\
$\widehat{\bar{Y}}_{M 2}$ & 115.99 & 122.51 & 128.28 \\
$\widehat{\bar{Y}}_{M 3}$ & 314.25 & 242.94 & 205.63 \\
$\widehat{\bar{Y}}_{M 4}$ & 29.27 & 29.49 & 29.66 \\
$\widehat{\bar{Y}}_{M 5}$ & 367.93 & 289.48 & 247.32 \\
$\widehat{\bar{Y}}_{M 6}$ & 115.66 & 122.02 & 127.63 \\
$\widehat{\bar{Y}}_{M 7}$ & 321.82 & 251.66 & 214.31 \\
$\widehat{\bar{Y}}_{M 8}$ & 329.78 & 267.25 & 232.08 \\
$\widehat{\bar{Y}}_{M 9}$ & 114.77 & 120.70 & 125.91 \\
$\widehat{\bar{Y}}_{M 10}$ & 321.50 & 260.02 & 225.54 \\
$\widehat{\bar{Y}}_{S K}$ & 314.68 & 258.16 & 225.75 \\
$\widehat{\bar{Y}}_{P}$ & 401.11 & 308.73 & 260.72 \\
$\widehat{\bar{Y}}_{M}$ & 412.55 & 315.18 & 265.15 \\
\hline
\end{tabular}

if

$$
\begin{aligned}
& \left(\lambda-\lambda^{\prime}\right) C_{y}^{2}\left\{\frac{\left(\rho_{y u}-\rho_{y x} \rho_{x u}\right)^{2}}{1-\rho_{x u}^{2}}\right\} \\
& +\lambda^{*} C_{y(2)}^{2}\left\{\frac{\left(\rho_{y u(2)}-\rho_{y x(2)} \rho_{x u(2)}\right)^{2}}{1-\rho_{x u(2)}^{2}}\right\}>0 .
\end{aligned}
$$

(ii) Further $\widehat{\bar{Y}}_{M}$ will be more efficient than $\widehat{\bar{Y}}_{P}$; that is,

$$
M\left(\widehat{\bar{Y}}_{M}\right)_{\mathrm{opt}}<M\left(\hat{\bar{Y}}_{P}\right)_{\mathrm{opt}}
$$

if

$$
\begin{aligned}
(\lambda- & \left.\lambda^{\prime}\right) C_{y}^{2}\left\{\frac{\left(\rho_{y u}-\rho_{y x} \rho_{x u}\right)^{2}}{1-\rho_{x u}^{2}}\right\} \\
& +\lambda^{*} C_{y(2)}^{2}\left\{\frac{\left(\rho_{y u(2)}-\rho_{y x(2)} \rho_{x u(2)}\right)^{2}}{1-\rho_{x u(2)}^{2}}\right\}>0 .
\end{aligned}
$$

Equations (37) and (39) are obviously true.

\section{Empirical Study}

We use the following data set for comparison.

5.1. Source (See [18]). Let $y$ be the output of the factory and let $x$ be the number of workers working in the factory. We randomly select a sample of size 20 from population of size 80 and considered this as the stratum of nonrespondents. For this population, we have $N=80, N_{2}=20, n=30$, $n_{2}=12, \bar{Y}=5182.6, \bar{X}=285.13, \bar{U}=153514, S_{y}^{2}=$ $3369642, S_{x}^{2}=73132.1, S_{u}^{2}=66013595417, S_{y(2)}^{2}=2800048$, $S_{x(2)}^{2}=76595.88, S_{u(2)}^{2}=68803364254, S_{y x}=454211$, $S_{y u}=380054066, S_{x u}=66781955, S_{y x(2)}=437594.9$, $S_{y u(2)}=381467001, S_{x u(2)}=70737846, \rho_{y x}=0.915$, $\rho_{y u}=0.806, \rho_{x u}=0.961, \rho_{y x(2)}=0.9449, \rho_{y u(2)}=0.8691$, $\rho_{x u(2)}=0.99744$.

\section{Discussion of Results}

The results based on above data set are given in Tables 3, 4 and 5. For $p=1$ in (13), the estimator $\widehat{\bar{Y}}_{P}$ reduces to the Singh and Kumar [13] estimator and for $p=1$ in (13), both $\widehat{\bar{Y}}_{P}$ and $\widehat{\bar{Y}}_{M}$ also have the same mean square error equal to mean square error of Singh and Kumar [13] estimator. From efficiency point of view the performances of all the ratio type estimators given in Table 3 are poor for $p=0.8,1$. Further the estimators, $\widehat{\bar{Y}}_{\mathrm{SK}}, \widehat{\bar{Y}}_{P}$, and $\widehat{\bar{Y}}_{M}$ (for optimum value), are equally efficient for $p=1$. Efficiency of the considered estimators decreases with an increase in inverse sampling rate $k$, except $\widehat{\bar{Y}}_{M 1}, \widehat{\bar{Y}}_{M 5}$, and $\widehat{\bar{Y}}_{M 8}$ where it increased.

Further for $p=0.8,0.5,0.35,0.3$, efficiency of considered estimators decreases with an increase in inverse sampling rate $k$ but for $\widehat{\bar{Y}}_{M 2}, \widehat{\bar{Y}}_{M 6}$, and $\widehat{\bar{Y}}_{M 9}$ (as they deal with the case when there is incomplete information on the study variable and complete information on the auxiliary variable), it increased. Efficiency increased dramatically for all considered ratio type estimators for values of $p<1$. As the value of $p$ decreases, efficiency increases and reaches the maximum at $p=0.35$ and then again starts decreasing. The performance of the suggested class of estimators $\widehat{\bar{Y}}_{P}$ and $\widehat{\bar{Y}}_{M}$ is better than all considered estimators for different combination of $k$ and $p$. The estimator $\widehat{\bar{Y}}_{M}$ is the best among all considered estimators and is preferable to use it.

\section{Conclusion}

In the present study auxiliary information has been used for enhancing the efficiency of estimators of finite population mean. Ratio, product, regression, and modification of these estimators are example of estimators which utilize auxiliary information. It is well established that ratio and product estimators are conditionally relatively more efficient than mean per unit estimator. Using fractional raw moments of auxiliary variable helps relaxing the efficiency condition and as a result the efficiency of ratio and product type estimators increases in the presence of nonresponse. The relative efficiency of the estimators of finite population mean can further be increased by making a combined use of fractional raw moments and mean of the auxiliary variable. We suggest using fractional raw moments of auxiliary variable, if available, in order to estimate the finite population mean more precisely. 


\section{Conflict of Interests}

The authors declare that there is no conflict of interests regarding the publication of this paper.

\section{Acknowledgment}

This project was funded by the Deanship of Scientific Research (DSR) at King Abdulaziz University, Jeddah, under Grant no. 130-212-D1435. The authors, therefore, acknowledge with thanks DSR technical and financial support.

\section{References}

[1] M. H. Hansen and W. N. Hurwitz, "The problem of nonresponse in sample surveys," Journal of the American Statistical Association, vol. 41, no. 236, pp. 517-529, 1946.

[2] W. G. Cochran, Sampling Techniques, John Wiley \& Sons, New York, NY, USA, 3rd edition, 1977.

[3] P. S. R. S. Rao, "Ratio estimation with sub sampling the nonrespondents," Survey Methodology, vol. 12, pp. 217-230, 1986.

[4] P. S. R. S. Rao, "Ratio and regression estimates with subsampling the non respondents," in Proceedings of the International Statistical Association Meetings, Tokyo, Japan, September 1987.

[5] B. B. Khare and S. Srivastava, "Estimation of population mean using auxiliary character in the presence of non-response," National Academy of Science Letters India, vol. 16, pp. 111-114, 1993.

[6] B. B. Khare and S. Srivastava, "Study of conventional and alternative two-phase sampling ratio, product and regression estimators in prese nce of non-response," Proceedings of the Indian National Science Academy, vol. 65, pp. 195-203, 1995.

[7] B. B. Khare and S. Srivastava, "Transformed ratio type estimators for the population mean in the presence of nonresponse," Communications in Statistics: Theory and Methods, vol. 26, no. 7, pp. 1779-1791, 1997.

[8] F. C. Okafor and H. Lee, "Double sampling for ratio and regression estimation with sub sampling the non-respondent," Survey Methodology, vol. 26, pp. 183-188, 2000.

[9] R. Tabasum and I. A. Khan, "Double sampling for ratio estimation with non-response," Journal of the Indian Society of Agricultural Statistics, vol. 58, no. 3, pp. 300-306, 2004.

[10] R. Tabasum and I. A. Khan, "Double sampling ratio estimator for the population mean in presence of non-response," Assam Statistical Review, vol. 20, pp. 73-83, 2006.

[11] A. A. Sodipo and K. O. Obisesan, "Estimation of the population mean using difference cum ratio estimator with full response on the auxiliary character," Research Journal of Applied Sciences, vol. 2, pp. 769-772, 2007.

[12] H. P. Singh and S. Kumar, "A regression approach to the estimation of the finite population mean in the presence of nonresponse," Australian \& New Zealand Journal of Statistics, vol. 50, no. 4, pp. 395-408, 2008.

[13] H. P. Singh and S. Kumar, "A general procedure of estimating the population mean in the presence of non-response under double sampling using auxiliary information," Statistics and Operations Research Transactions, vol. 33, no. 1, pp. 71-83, 2009.

[14] H. P. Singh and S. Kumar, "Estimation of mean in presence of non-response using two phase sampling scheme," Statistical Papers, vol. 51, no. 3, pp. 559-582, 2010.
[15] H. P. Singh, S. Kumar, and M. Kozak, "Improved estimation of finite-population mean using sub-sampling to deal with non response in two-phase sampling scheme," Communications in Statistics: Theory and Methods, vol. 39, no. 5, pp. 791-802, 2010.

[16] V. Dubey and M. Uprety, "A class of estimators of population mean using auxiliary information in the presence of nonresponse," Model Assisted Statistics and Applications, vol. 3, no. 2, pp. 119-125, 2008.

[17] A. Y. Al-Hossain and M. Khan, "Efficiency of ratio, product, and regression estimators under maximum and minimum values, using two auxiliary variables," Journal of Applied Mathematics, vol. 2014, Article ID 693782, 6 pages, 2014.

[18] M. N. Murthy, Sampling Theory and Methods, Statistical, Calcutta, India, 1967. 


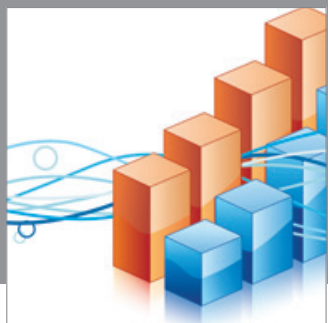

Advances in

Operations Research

mansans

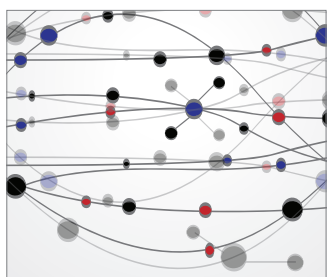

The Scientific World Journal
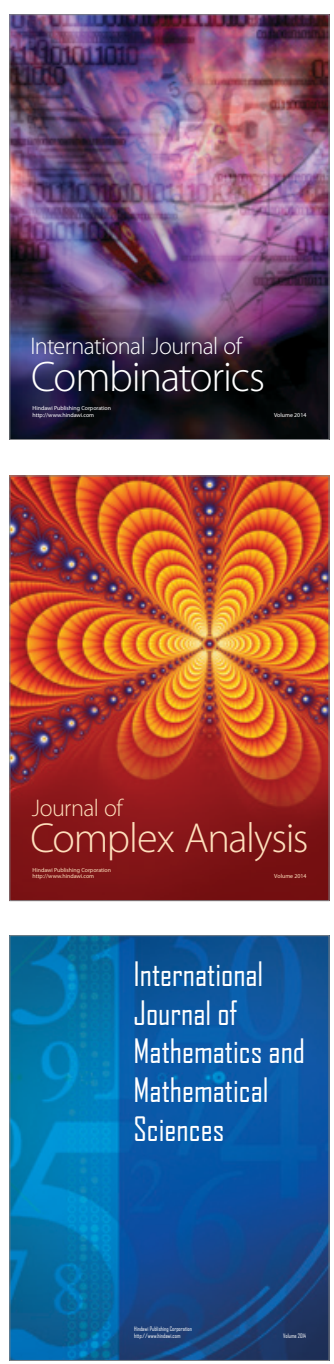
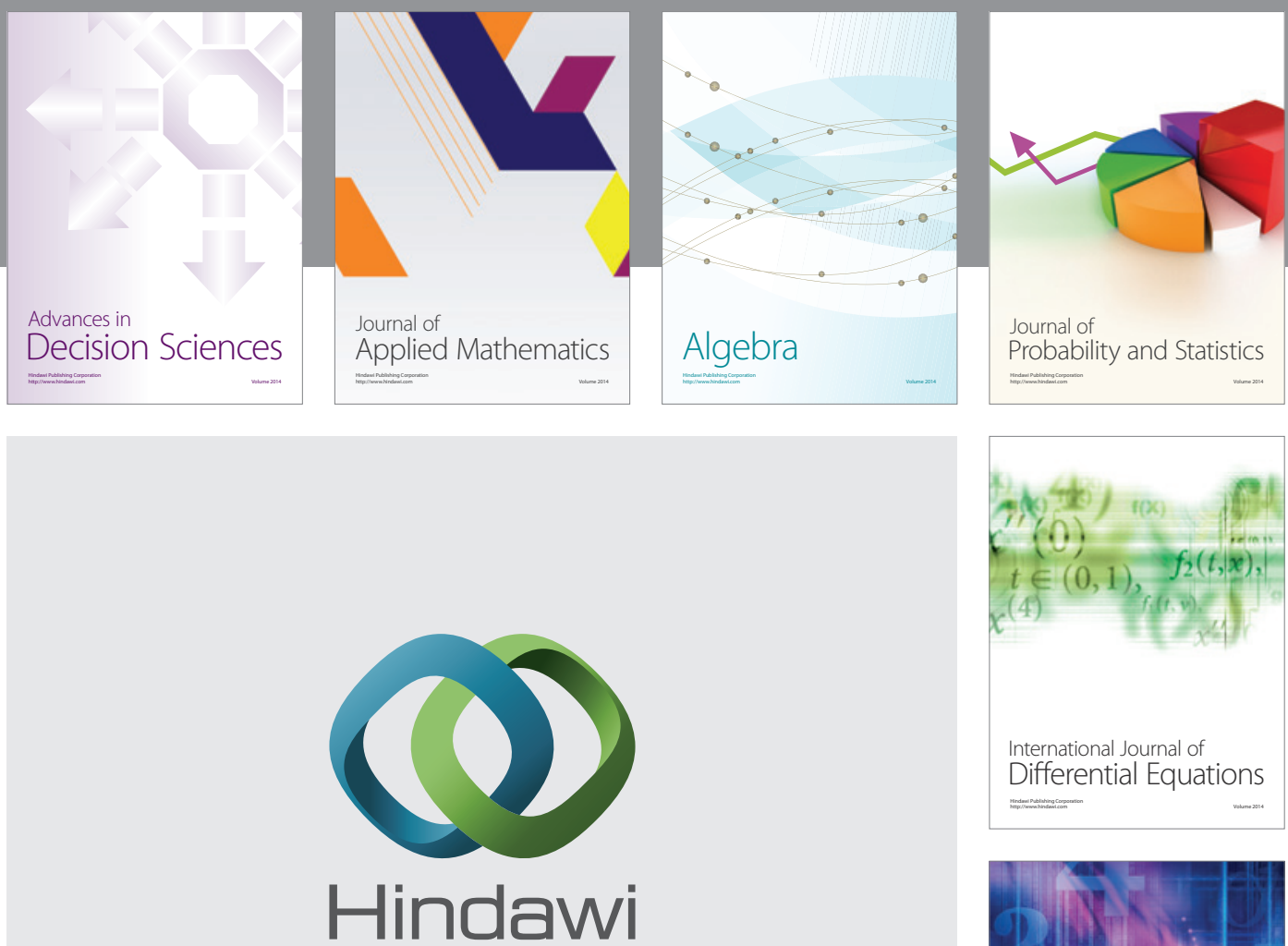

Submit your manuscripts at http://www.hindawi.com
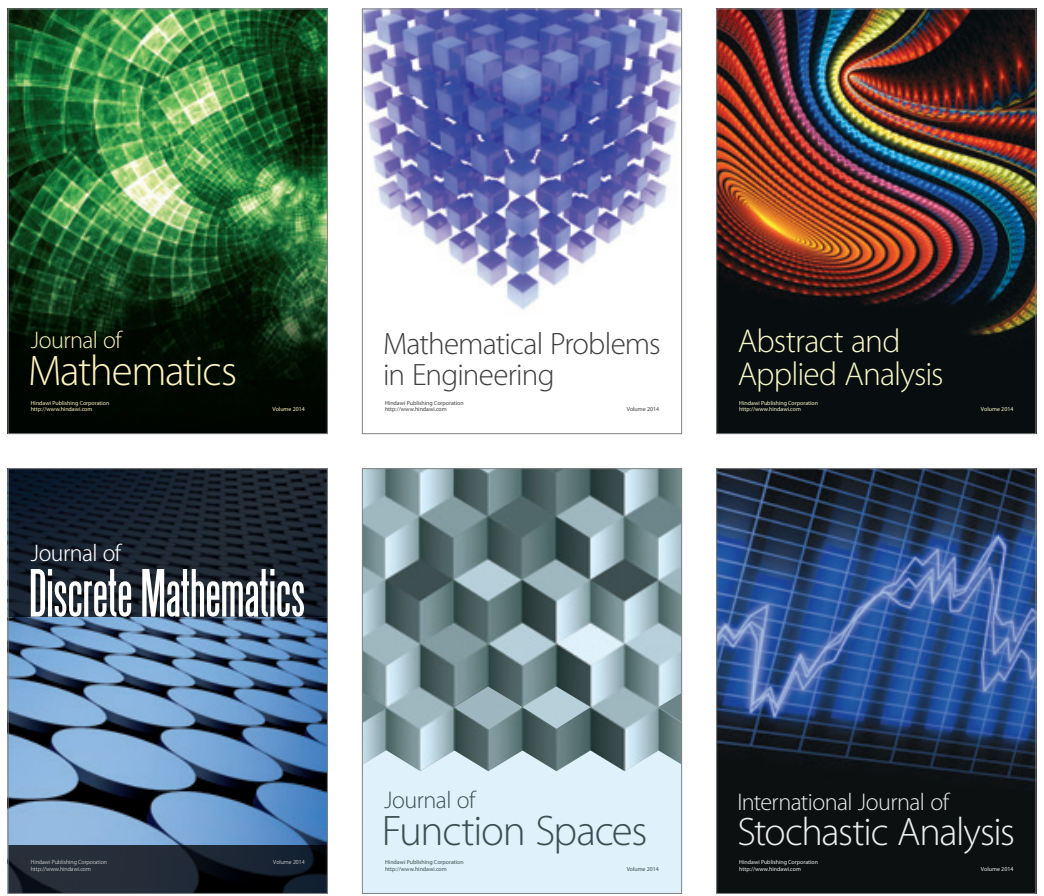

Journal of

Function Spaces

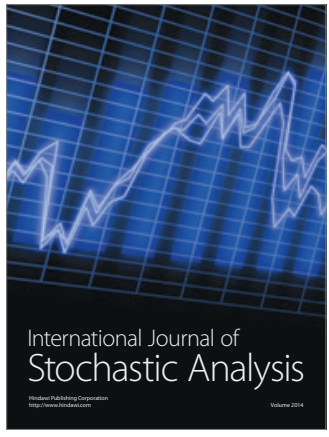

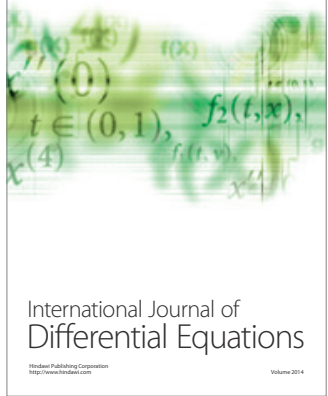
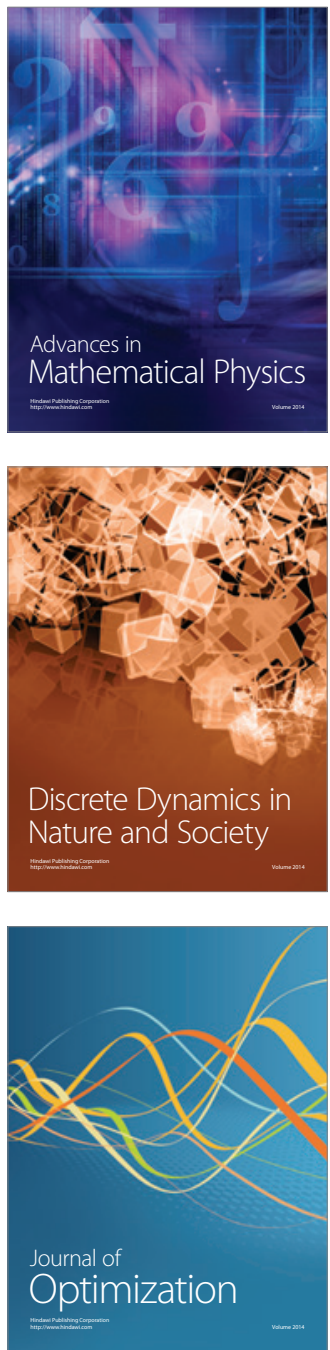